\title{
Competence in confirming correct placement of nasogastric feeding tubes amongst FY1 doctors
}

\author{
Sindy Lee, Eleanor Mason
}

\begin{abstract}
Foundation year one (FY1) doctors are required to confirm correct placement of nasogastric (NG) feeding tubes on chest radiography on a regular basis. Many FY1s do not receive formal training during medical school or during the FY1 year. Multiple incidents of harm to patients, including death, resulting from incorrect placement of an NG feeding tube have been reported to the National Patient Safety Agency (NPSA) since 2005. Our audit assessed the confidence and knowledge of FY1 doctors in correctly confirming NG placement using a qualitative questionnaire. The results showed that both confidence and knowledge on the topic were remarkably poor. Our intervention consisted of a teaching session to the FY1 cohort, which included information on NPSA guidelines for identifying correct NG placement. A post-teaching questionnaire was attained from the same group of FY1s. Confidence and knowledge of the criteria had significantly improved. We concluded that there is clearly a need for teaching on this subject at an undergraduate and post-graduate level. The interactive peer-led style of our teaching session was shown to be highly effective. The addition of handouts and posters in the clinical workplace acted as helpful reminders for junior doctors to use at the point of need. Our project has highlighted a gap in training which poses a serious potential risk to patient safety. We have created a simple solution which, if adopted on a local and national scale, will facilitate a reduction in avoidable harm to patients.
\end{abstract}

\section{Problem}

Enteral feeding via nasogastric (NG) tube occurs frequently in inpatients of all age groups. The majority of NG tubes are inserted and used successfully without incident.(1) However, there is a risk of the tube being inserted into the respiratory tract or becoming misplaced out of the stomach after the initial insertion. To reduce subsequent harm to patients when this occurs, a National Patient Safety Agency (NPSA) alert was issued in 2005.(1) Despite this, a significant number of cases of harm have since been reported.(2)

The types of harm to patients included aspiration pneumonia, pulmonary haemorrhage, pneumothorax and death. Deaths due to NG misplacement have been identified as, "never events," by the Department of Health.(3)

Foundation year one (FY1) doctors are often asked to review chest X-rays (CXR) following NG tube insertion to confirm correct placement. Very few medical schools incorporate teaching on this topic into their curriculum, despite the importance of this skill in clinical practice as a foundation doctor. There is also usually no formal training during the FY1 year. The combination of the potential for serious harm and the lack of formal teaching represented a significant problem requiring intervention.

\section{Background}

Generally NG feeding tubes are used on a relatively short term basis in the hospital setting.(4) Nutritional support guidelines have indicated that any patient who is malnourished or at risk of malnutrition may require feeding via NG tube if there is "inadequate or unsafe oral intake" and a "functional, accessible gastrointestinal tract".(5) If an NG tube is inserted into the wrong place or if it becomes displaced, intrapulmonary feeding may occur. This can lead to significant, and potentially fatal, complications such as aspiration pneumonia.(4) Since 2005, there have been 79 cases of harm caused to patients from misplaced NG tubes.

Misinterpretation of CXR images was the most common cause of harm accounting for 45 serious incidents.(4) Twenty-one cases of death following incorrect NG placement have been reported since 2005,12 of these owing to incorrect analysis of the NG position on CXR.(2) Harm arising from the misinterpretation of NG tube position on CXR has been identified by the Department of Health as a "never event" as it should be entirely avoidable.(4)

Consequently, in March 2011, the NPSA issued another safety alert which focused on the safe confirmation of NG tube placement using CXR. This patient safety alert updated and reinforced the 2005 guidelines in an effort to minimise risk to patients, as well as to ensure that the guidelines were being reflected in the clinical domain and in trust policies.(3) The alert from 2011 states that documentation of the tube placement checking process is essential. The documentation must demonstrate the following:

1. Confirmation that the x-ray used was the most recent x-ray;

2. The criteria used for interpretation;

3. Instructions as to any required actions.(2)

The alert also recommended that healthcare professionals involved in confirming NG placement should have both theoretical and practical training.(3)

A similar audit in 2010 of junior doctors by the NPSA has shown a large variation in knowledge of the above guidance, including a lack of awareness of the guidelines and a lack of formal training in x-ray interpretation of NG placement.(4) There is therefore a need for intervention at a local, trust-based level, directed at FY1 doctors at 
the start of their medical career.

\section{Baseline Measurement}

This project assessed the competence in confirming NG tube position by CXR interpretation amongst FY1 doctors at Brighton and Sussex University Hospitals (BSUH). The assessment involved testing the FY1s' baseline knowledge of the NPSA guidelines and documentation of NG tube checks.

A questionnaire was given out to the 2012-13 cohort of FY1s at BSUH. The survey ascertained copious information about NG tube placement checking by FY1s, including whether it is a commonly performed task and their confidence in executing this task. In addition, the level of pre-existing knowledge about the national guidelines for checking NG tube placement was assessed, including the criteria for confirming correct placement on CXR.

Documentation of NG tubes placement checks in medical notes was also reviewed. The presence of the four criteria for confirming correct position on CXR was recorded, i.e. 1. NG tube descends centrally, 2. bisects the carina, 3. crosses the diaphragm in the midline, and 4. deviates to the left. All patients at the Royal Sussex County Hospital with NG feeding tubes during the period 14-18th January 2013 were included.

Baseline Results:

Almost all FY1s (88\%) had been asked by nursing staff to check the positioning of NG tubes. However, formal training on this skill was not included as part of the foundation teaching programme at the trust, with only $12 \%$ of the cohort having received prior training at medical school. Reflecting this lack of training, most FY1s did not feel very confident in performing NG tube checks (confidence was generally rated within the range of 5-8/10).

Knowledge of the first and second line methods for checking NG tube placements was good, with two thirds of the cohort correctly identifying $\mathrm{pH}$ sensitive paper and CXR respectively. Crucially, there was very poor knowledge of the $\mathrm{pH}$ measurements which necessitate the second line method to be employed ( $3 \%$ answered correctly), and most of the cohort (82\%) did not know the criteria for confirming placement on CXR.

Documentation in the medical notes was often poor and lacked sufficient detail. Twenty-eight percent of entries simply stated that the NG tube was, "safe to use", with $29 \%$ "in the stomach", or, "in situ", and $14 \%$ "below the diaphragm". No entries included all four criteria listed above.

See supplementary file: ds2162.docx - "FY1 Questionnaire for checking NG placement"

\section{Design}

Checking NG tube positioning is a routine task performed by FY1 doctors. Results from this audit demonstrate that knowledge of the guidelines in general, and the specific criteria for confirming positioning on CXR in particular, was patchy and remarkably poor in view of the potential for serious consequences of feeding through a misplaced NG tube. Clearly, there was room for improvements in both knowledge regarding relevant guidelines and in more detailed documentation.

Our intervention consisted of a teaching session for all FY1 doctors. This interactive session explained the NPSA guidelines and illustrated the four criteria to review on CXR. Teaching was also provided on ways to improve documentation of NG tube checks. In order to consolidate learning, an e-learning module providing further information and a self-assessment was strongly recommended to the group (www.trainingngt.co.uk). A summary of the guidelines was then given as a handout for use as a reference tool. Posters were placed in clinical areas to remind doctors of the guidelines and the criteria for doctors to use at the time of checking a CXR.

\section{Strategy}

Our strategy for addressing the insufficient knowledge of FY1 doctors on this topic was to provide a teaching session to the whole cohort of FY1s at our trust. This interactive teaching session summarised the NPSA guidelines. It also demonstrated the criteria for confirming correct positioning on CXR, as well as including examples of incorrectly placed NG tubes.

By highlighting the concerning statistics involving cases of patient harm from incorrect checking of NG tubes, in addition to the level of ignorance among junior doctors in the criteria for confirming placement, we were able to successfully engage our peers in the teaching session.

Feedback from FY1s was very positive; everyone found the teaching to be helpful and reported that they took something away from the session which they intend to implement in clinical practice.

In order to aid the retention of knowledge after the teaching session, handouts were provided which summarised the guidelines and posters were displayed in clinical areas to serve as reminders of the criteria for checking correct NG placement on CXR.

A re-audit took place five months after the initial audit to assess the impact of our interventions. A second questionnaire was given out to the same cohort of FY1s to test retention of knowledge from the teaching. The survey asked FY1s again to rate their confidence in checking NG tube positions. It also tested their knowledge of the first and second line tests, $\mathrm{pH}$ cut off and the criteria on CXR for confirming correct position of NG tubes.

\section{Results}

The following questions were asked in the post-teaching questionnaire:

1: How confident do FY1 doctors feel about confirming correct NG placement after attending the teaching session? 
- $20 \%$ of $\mathrm{FY} 1$ s rated $10 / 10$ in confidence

- $28 \%$ of $F Y 1$ s rated $9 / 10$ in confidence

- $32 \%$ of $F Y 1$ s rated $8 / 10$ in confidence

$-16 \%$ of $F Y 1 s$ rated $7 / 10$ in confidence

- $4 \%$ of $F Y 1 s$ rated $5 / 10$ in confidence

2: What is the first-line method for checking NG tube placement?

$80 \%$ of $\mathrm{FY} 1 \mathrm{~s}$ answered correctly, compared with $64 \%$ in the preteaching survey.

3: What is the second line method for checking NG placement?

$88 \%$ answered correctly, compared with $67 \%$ in the pre-teaching survey.

4: What is the recommended cut off $\mathrm{pH}$ for confirming gastric placement of NG tube?

$32 \%$ answered correctly, compared with $3 \%$ in the pre-teaching survey.

5: What are the criteria that should be used to confirm correct placement of NG tube on chest x-ray?

- $36 \%$ knew all four criteria (3\% pre-teaching)

- $56 \%$ knew the first criterion ( $8 \%$ pre-teaching)

- $44 \%$ knew the second criterion (0\% pre-teaching)

- $88 \%$ knew the third criterion ( $48 \%$ pre-teaching)

- $68 \%$ knew the fourth criterion (26\% pre-teaching)

Confidence among FY1s in performing NG tube checks ranged from mostly $5-8 / 10$ before the audit and generally $8-10 / 10$ after the intervention. No FY1s rated themselves as 9 or 10 at baseline; this compares with $48 \%$ of the year reporting a 9 or 10 rating after the teaching.

Knowledge of the guidelines for confirming NG tube placement also improved. There was an increase in the percentage of people correctly identifying $\mathrm{pH}$ testing and $\mathrm{CXR}$, as the first and second line tests, respectively. Around one third of FY1s identified pH 5.5 as the cut off for correct placement of NG tubes, compared with only $3 \%$ prior to our teaching session.

With regards to the specific criteria to look for on CXR, there was an obvious improvement in the ability of FY1s to list the four criteria previously outlined. One third of the cohort could list all four criteria, compared with only $3 \%$ before the audit.

Our post-intervention questionnaire, five months after the initial audit, demonstrates a global improvement in knowledge and confidence in confirming NG tube position on CXR amongst FY1s at BSUH.

See supplementary file: ds2163.ppt - "Percentage of FY1 doctors who know the criteria for confirming correct NG placement on chest x-ray"

\section{Lessons and Limitations}

Our idea of providing a succinct teaching session at the weekly FY1 teaching was a simple one. The practicalities of coordinating this session into a busy timetable proved to be more difficult. It required liaising with the foundation programme administrator to choose a suitable slot and was contingent on other presentations of the day running on time.

The feedback received from colleagues was very positive. Our FY1 colleagues found the teaching to be helpful and relevant to clinical practice. In order to ensure sustainability of our intervention, we were very keen for the next cohort of 2013-14 FY1s to also receive similar training on checking NG tubes.

Ideally, we would have liked to incorporate a teaching session into the Foundation Curriculum at the trust and to include the e-learning module into the FY1 induction programme. However we were informed that the induction programme was already full for the coming year and adding an e-learning module to the induction programme requires backing and funding from a named department which we did not have.

We learned that implementing change to clinical practice can be a process which takes a longer time to organise and coordinate than previously anticipated. In addition, support from named consultants and departments interested in the project would help to drive change. Finally, finding the appropriate teams and contacts to liaise with in the first instance is crucial for encouraging interest and galvanising change - an essential step for taking any project forward.

\section{Conclusion}

Complications from misplaced NG tubes are avoidable and death arising from introducing feed into the lungs is a "never event". Given the potential for serious harm and risk to patient safety, confirmation of NG tube positioning must only be carried out by those competent in this skill.

Our audit highlighted a gap in teaching at both undergraduate and post-graduate levels of medical training, as evidenced by the poor knowledge amongst FY1s in the formal guidelines and criteria for confirming correct NG tube placement.

A short, interactive peer-led teaching session is an effective method for improving confidence and knowledge among FY1s in confirming correct NG tube position on CXR. Teaching can be reinforced with summary handouts, posters in clinical areas and an invitation to 
complete an e-learning module. Other Trusts may also benefit from incorporating training in NG tube checking into their Foundation Programme curricula.

Area for further work

Although we have shown improvement in knowledge with our intervention, there is an opportunity for further work at the local level to ensure sustainability for future cohorts. We have identified the following areas for consideration:

- Inclusion of an annual teaching session on correct placement of NG feeding tubes into the FY1 curriculum at local trusts.

- Inclusion of guidelines on correct placement of NG feeding tubes on the Trust intranet and in handbooks for junior doctors.

- Encouragement by the foundation programme director for new FY1 doctors to complete an e-learning module.

\section{References}

1. National Patient Safety Agency. Reducing harm caused by the misplacement of nasogastric feeding tubes; Patient Safety Alert. [Internet]. 2005 Feb [cited 2013 June 14]. Available from: www.nrls.npsa.nhs.uk/resources/?entryid45=59794

2. National Patient Safety Agency. Reducing the harm caused by misplaced nasogastric feeding tubes in adults, children and infants. Patient Safety Alert. [Internet]. 2011 Mar [cited 2013 June 23]. Available from: www.nrls.npsa.nhs.uk/resources/type/alerts/?entryid45=129 $\underline{640}$

3. Nutrition Support Team, Salford Royal NHS Foundation Trust. Nasogastric Feeding Tube Placement and Management Resource Manual. [Internet] 2011 Aug [cited 2013 Jul 10]. Available from: http://elearning.hopeacademic.org.uk/srht_elearn_dept/

4. Lamont T, Beaumont C, Fayaz A, Healey F, Huehns T, Law R, Lecko C, Panesar S, Surkitt-Parr M, Stroud M, Warner B. Checking placement of nasogastric feeding tubes in adults (interpretation of $x$ ray images): summary of a safety report from the National Patient Safety Agency. BMJ. 2011;342:d2586

5. National Institute for Clinical Excellence. Nutrition support for adults - oral nutrition support, enteral tube feeding and parenteral nutrition. [Internet]. 2006 Feb [cited 2013 June 23]. Available from: www.nice.org.uk/nicemedia/live/10978/29978/29978.pdf

\section{Declaration of interests}

Nothing to declare

\section{Acknowledgements}

None 